\title{
Prescribing Patterns and Patient Outcomes for Bone and Joint Infections Treated with Cefazolin and Probenecid: A Retrospective Observational Study
}

\author{
Marci E Dearing, Sarah V Burgess, Valerie Murphy, Samuel Campbell, Lynn Johnston, \\ and Tasha D Ramsey
}

\begin{abstract}
Background: Previous studies have described the use of cefazolin with probenecid to treat uncomplicated skin and soft-tissue infections. Some prescribers are extrapolating from this evidence to treat more invasive infections, which have a greater potential for poor outcomes, including treatment failure that could lead to increased morbidity and mortality. Information supporting cefazolin with probenecid as effective treatment in this context is needed.
\end{abstract}

Objectives: To describe prescribing patterns and outcomes for patients who received cefazolin with probenecid for the treatment of bone and joint infections.

Methods: This single-centre retrospective study involved adult outpatients for whom cefazolin and probenecid were prescribed for bone and joint infections between April 1, 2012, and March 31, 2017. Patient charts were reviewed, and data were collected for clinical and microbiological variables using a standardized data collection form.

Results: In a total of 80 cases, the patient received cefazolin and probenecid for treatment of a bone or joint infection, of which 69 cases met the inclusion criteria. In most cases $(n=67)$, the patients were treated with cefazolin $2 \mathrm{~g}$ IV plus probenecid $1 \mathrm{~g}$ PO, both given twice daily. Completion of prescribed treatment occurred in 56 patient cases (81\%), resolution of signs and symptoms in $53(77 \%)$, readmission to hospital in $11(16 \%)$, recurrence of infection in $6(9 \%)$, and treatment failure requiring a change in therapy in $7(10 \%)$.

Conclusions: The effectiveness of cefazolin and probenecid for the treatment of bone and joint infections appears to be similar to that of standard treatment, as reported in the literature. Antibiotic effectiveness is difficult to determine conclusively in a retrospective analysis, so these results should be interpreted with caution, but they may stimulate further research.

Keywords: cefazolin, probenecid, bone infection, joint infection, outpatient parenteral antimicrobial therapy

\section{RÉSUMÉ}

Contexte : Des études précédentes ont décrit l'utilisation de la céfazoline et du probénécide pour traiter les infections cutanées et les infections de tissus mous. Quelques prescripteurs extrapolent ces éléments probants pour traiter des infections plus invasives, dont les résultats risquent d'être défavorables, comme un échec du traitement pouvant entraîner une morbidité et une mortalité accrues. De l'information supplémentaire étayant l'efficacité du traitement à l'aide de la céfazoline et du probénécide dans ce contexte est nécessaire.

Objectifs : Décrire les modes de prescription et les résultats obtenus par des patients ayant reçu de la céfazoline et du probénécide pour traiter des infections osseuses et articulaires.

Méthodes : Cette étude rétrospective unicentrique porte sur des patients ambulatoires adultes à qui on a prescrit de la céfazoline et du probénécide pour traiter des infections osseuses et articulaires entre le $1^{\text {er }}$ avril 2012 et le 31 mars 2017. L'examen des dossiers médicaux des patients a permis la récolte de données sur les variables cliniques et microbiologiques à l'aide d'un formulaire de recueil de données standard.

Résultats : Les patients, soit 80 cas en tout, ont reçu de la céfazoline et du probénécide pour traiter une infection osseuse ou articulaire et 69 de ces cas répondaient aux critères d'inclusion. Dans la plupart des cas $(n=67)$, les patients étaient traités avec de la céfazoline IV dosée à $2 \mathrm{~g}$ et du probénécide dosé à $1 \mathrm{~g}$ PO, les deux produits étant administrés deux fois par jour. Le traitement a été appliqué au complet dans 56 cas (81\%), la résolution des signes et des symptômes a eu lieu dans 53 cas (77\%), la réadmission à l'hôpital s'est produite dans 11 cas (16\%), les infections ont récidivé dans 6 cas $(9 \%)$ et le traitement s'est soldé par un échec et a nécessité un changement de thérapie dans 7 cas (10\%).

Conclusions : L'efficacité de la céfazoline et du probénécide dans le traitement des infections osseuses et articulaires semble être similaire à celle des traitements standard, comme le rapporte la littérature scientifique. L'efficacité des antibiotiques est difficile à déterminer de façon concluante dans une analyse rétrospective, ces résultats doivent donc être interprétés avec prudence, mais ils pourraient stimuler des recherches supplémentaires.

Mots-clés : céfazoline, probénécide, infection osseuse, infection articulaire, thérapie antimicrobienne parentérale des patients ambulatoires 


\section{INTRODUCTION}

$\mathrm{M}$

anagement of bone and joint infections can be challenging, as there are many different approaches to treatment, and outcomes may be poor, with significant complications and prolonged courses of antibiotics. ${ }^{1}$ In an era when antimicrobial resistance, health care spending, and hospital capacity are serious public health issues, treatment strategies that address these concerns are paramount. ${ }^{2}$ A treatment approach to bone and joint infection that takes into consideration antimicrobial stewardship, health care resources, and patient outcomes is essential.

Treatment of infection with cefazolin and probenecid was first described in the $1970 \mathrm{~s} .{ }^{3,4}$ Cefazolin, traditionally given every $8 \mathrm{~h}$ for infections requiring IV treatment, is a narrow-spectrum antibiotic that is preferred for treating many pathogens implicated in skin and soft-tissue infections, as well as bone and joint infections. Probenecid, an oral uricosuric agent with no antimicrobial activity, impairs the renal excretion of cefazolin, thus extending its half-life. ${ }^{5}$ A challenge for outpatient parenteral antimicrobial therapy, when the availability of home IV infusion pumps is limited, is balancing selection of an antimicrobial that has convenient dosing (such as once-daily ceftriaxone or ertapenem) but a broader-than-necessary spectrum of activity with antimicrobial stewardship. ${ }^{2}$ Coadministration of cefazolin with probenecid allows once- or twice-daily administration and improves the suitability of cefazolin for use as outpatient parenteral antimicrobial therapy.,

The evidence for the combination of cefazolin and probenecid is limited to the treatment of skin and soft-tissue infections and gonorrhea; this drug combination has not been evaluated for other infections. ${ }^{3,4,6,7}$ Nonetheless, extrapolation of the available evidence and pharmacokinetic principles has prompted some clinicians to use cefazolin with probenecid for other types of infections with susceptible pathogens. This combination has been prescribed at our institution for outpatient treatment of osteomyelitis, diskitis, septic arthritis, and prosthetic joint infections. These infections, most commonly caused by Staphylococcus aureus, are associated with high rates of relapse and recurrence. ${ }^{8,9}$ They represent a significant burden to the health care system and require prolonged treatment with antimicrobials. ${ }^{10}$ Inadequate treatment can result in devastating complications, such as loss of limb function, amputation, bone loss, and death. ${ }^{8-10}$

Outcomes related to osteomyelitis are challenging to study, in part because of the diverse nature of the infection. ${ }^{9}$ Some researchers recommend against using the term "cure" because of the inherently high recurrence rate and the possibility of chronic infection, which make it difficult to determine treatment effectiveness. ${ }^{8}$ A 2013 Cochrane systematic review estimated the long-term recurrence rate for osteomyelitis at approximately $20 \%$. $^{11}$ Treatment failure rates for vertebral osteomyelitis have ranged from $10 \%$ to $30 \%$ in clinical trials. ${ }^{12}$ One study reported recovery from septic arthritis in 53\% to 69\% of treated patients, depending on the treatment modality. ${ }^{13}$ For prosthetic joint infections, treatment success rates ranged from $31 \%$ to $82 \%$ for prosthetic retention and debridement to $90 \%$ for 2 -stage exchange procedures. $^{14}$

The purpose of this study was to describe prescribing patterns and outcomes for patients who received cefazolin with probenecid as outpatient therapy for the treatment of osteomyelitis (including the vertebral form), diskitis, septic arthritis, and prosthetic joint infections either following discharge from hospital or in the emergency department of the Queen Elizabeth II Health Sciences Centre in Halifax, Nova Scotia.

\section{METHODS}

\section{Study Design}

This single-centre, retrospective, observational study utilized a chart review to collect information about patients' demographic and clinical characteristics. The chart review was approved as a quality assurance project by the Nova Scotia Health Authority Research Ethics Board, and the requirement for patient informed consent was waived.

\section{Patient Population and Screening}

The population of interest consisted of patients who received cefazolin and probenecid as outpatient therapy for treatment of osteomyelitis, diskitis, septic arthritis, or prosthetic joint infection. Databases within the hospital's electronic discharge medication reconciliation and pharmacy software (BDM Pharmacy, BDM IT Solutions Inc, Saskatoon, Saskatchewan) were searched for patients for whom cefazolin and probenecid were prescribed from April 1, 2012, to March 31, 2017.

\section{Inclusion and Exclusion Criteria}

The study included patients who received cefazolin and probenecid as outpatient therapy. Adult patients with osteomyelitis, diskitis, septic arthritis, or a prosthetic joint infection for whom cefazolin and probenecid were prescribed either while they were inpatients (as a test dose in preparation for home administration after discharge from the study hospital) or while they were outpatients receiving therapy in the emergency department, were eligible for inclusion. Patients were excluded if they were less than 18 years of age, if their home address and/or permanent residence was outside of Nova Scotia (which precluded follow-up after discharge), if they were discharged from hospital with antimicrobial therapy other than cefazolin and probenecid (because of susceptibility information that became available after the initial test dose of cefazolin and probenecid), or if cefazolin and probenecid were used to facilitate short-term treatment outside of hospital (e.g., a weekend pass) without prescription of a full course of therapy. 


\section{Outcome Measures}

The primary outcome measure was the percentage of patients who successfully completed the intended course of cefazolin and probenecid therapy. Success was defined as documented completion of the intended cefazolin and probenecid therapy, whether or not oral antibiotics were used after the IV course to complete the prescribed duration of therapy or for suppression of chronic infection.

Secondary outcome measures were the percentages of patients for whom cefazolin and probenecid therapy was initiated with the following characteristics:

- resolution of infection, defined as initial and sustained resolution of signs and symptoms, microbiological cure (if results were available), and no additional IV antimicrobial therapy for treatment of the bone and joint infection at 12 months from the end of the cefazolin and probenecid course $^{15}$

- readmission for inpatient antimicrobial therapy related to the bone or joint infection up to 12 months after completion of initial therapy ${ }^{15}$

- change in antibiotic therapy due to presumed treatment failure or recurrence of infection during the defined treatment course or initiated within 1 month after completion of cefazolin and probenecid (separate from step-down to planned oral therapy)

- all-cause mortality during cefazolin and probenecid treatment and up to 12 months after completing the cefazolin and probenecid treatment ${ }^{15}$
- step-down to oral antibiotics to complete the planned duration of therapy or for suppression of chronic infection

- adverse effect(s) of cefazolin and/or probenecid causing discontinuation and/or change in therapy during the defined treatment course

\section{Data Collection}

After eligible patients were identified, relevant data were collected from the scanned charts in the Horizon Patient Folder and Clinical Portal databases using a standard data collection form (see Appendix 1, available at https://www.cjhp-online.ca/ index.php/cjhp/issue/view/198/showToc). Information collected included demographic data, clinical characteristics, prescribing patterns for cefazolin and probenecid, and the outcomes of interest. Other antimicrobial therapy related to treatment of bone or joint infection and prescribed at the time of admission, while in hospital, or at the time of discharge was also recorded.

\section{Statistical Analysis}

Descriptive statistics were used to analyze and report the primary and secondary outcomes as a percentage or a mean with standard deviation (SD), as appropriate.

The association of specific outcome measures with baseline characteristics (age, sex, creatinine clearance, immunocompromise or immunocompetence, recurrent or chronic infection, specific type of bone or joint infection, location of prosthetic joint infection), additional antimicrobial therapy, empiric therapy (defined as either absence of microbiological culture or no
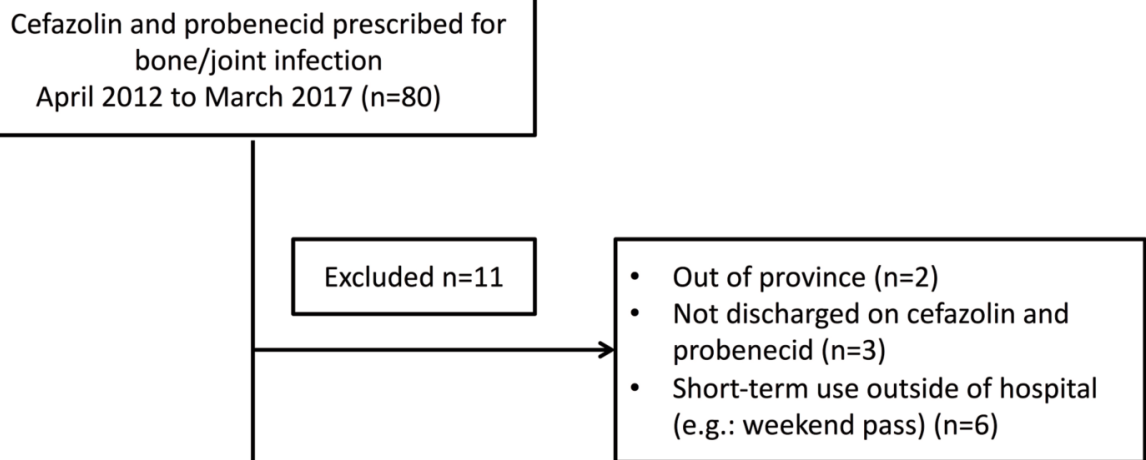

Figure 1. Flow diagram showing derivation of the study sample. The data ( $n$ values) are presented in terms of the number of patient cases (not number of patients), because 1 patient had 2 separate courses of therapy that qualified for inclusion. 
growth), and monomicrobial or polymicrobial etiology was analyzed using univariate exact logistic regression. Results are reported as unadjusted odds ratios (ORs) with 95\% confidence intervals (CIs) and associated $p$ values, with statistical significance defined as $p<0.05$. The analysis was completed using SAS statistical software, version 9.4 (SAS Institute, Cary, NC).

\section{RESULTS}

In a total of 80 patient cases, cefazolin and probenecid were prescribed for the treatment of a bone or joint infection. These cases represented a total of 79 patients, because 1 patient was treated on 2 separate occasions more than 12 months apart for infection in the same joint. Of the 80 patient cases, 69 met the inclusion criteria (Figure 1), including both treatment courses for the patient who had 2 treatments. Results are therefore presented in terms of patient cases (rather than patients) where appropriate. The baseline characteristics of the patient cases are presented in Table 1. Among the 69 patient cases, 33 (48\%) were male, and the mean age was 62.0 (SD 14.1) years. In nearly half of the cases $(n=34$ [49\%]), there was prosthetic material in the infected area, $24(35 \%)$ had a history of bone or joint infection, and $20(29 \%)$ had a recurrent or chronic infection. The most common treatment indication was osteomyelitis $(n=29$ [42\%]), and the most common pathogen was methicillin-susceptible $S$. aureus $(n=31[45 \%])$. Fourteen $(20 \%)$ of the patient cases were treated empirically. The mean duration of cefazolin and probenecid therapy was 36.0 (SD 16.1) days.

Almost all patient cases were treated with cefazolin $2 \mathrm{~g} \mathrm{IV}$ twice daily preceded by probenecid $1 \mathrm{~g}$ PO twice daily (typically given $30 \mathrm{~min}$ before) (Table 2). One patient with a prosthetic joint infection received cefazolin $1 \mathrm{~g}$ IV preceded by probenecid $1 \mathrm{~g}$ PO, both given twice daily, and 1 patient with osteomyelitis received cefazolin $2 \mathrm{~g}$ IV preceded by probenecid $1 \mathrm{~g}$ PO, both given once a day. The reason for these lower doses was not clear from the patient records.

The prescribed course of cefazolin and probenecid therapy was completed in 56 patient cases (81\%) (Table 3). Reasons for treatment not being completed as prescribed were intolerance, death, cefazolin resistance (as documented on susceptibility testing), patient's request for a once-daily regimen after starting treatment, recurrence of infection, and readmission to hospital (both related and unrelated to the infection) (Table 3). Prescribers determined the duration of cefazolin and probenecid treatment and the need for oral step-down therapy on the basis of clinical experience, patient factors, and infection characteristics (e.g., infection location or origin, pathogen, recurrence risk factors, presence of prosthetic material).

Step-down from IV to oral antibiotics occurred in 39 (57\%) of the patient cases. Among these 39 cases, treatment with oral therapy was completed in 33 (85\%) (for which mean duration of oral therapy was 61.5 days), and oral therapy was continued for
Table 1. Baseline Characteristics

$\begin{array}{lrc}\text { Characteristic } & \text { No. (\%) of Cases* } \\ (\boldsymbol{n}=\mathbf{6 9})\end{array}$

$\overline{\mathrm{BMI}}=$ body mass index (calculated as weight in kilograms divided by height in meters squared), MRSA = methicillin-resistant Staphylococcus aureus, MSSA = methicillin-susceptible Staphylococcus aureus, $\mathrm{SD}=$ standard deviation

*Except where indicated otherwise. The 69 patient cases represented a total of 68 patients; 1 patient had 2 separate courses of therapy that qualified for inclusion.

tRheumatic disease includes rheumatoid arthritis, osteoarthritis, ankylosing spondylitis, and psoriatic arthritis.

suppression of chronic infection in $6(15 \%)$. In most of these cases (27 [69\%] of 39), IV therapy was stepped down to oral cephalexin.

Initial resolution of signs and symptoms was documented in $53(77 \%)$ of all patient cases. In 49 (92\%) of these 53 cases, resolution was sustained for a period of 12 months after the end of cefazolin and probenecid treatment. Of the 4 patient cases 
Table 2. Cefazolin and Probenecid Prescribing Patterns and Related Treatment

\begin{tabular}{|c|c|}
\hline $\begin{array}{l}\text { Prescribing Patterns and } \\
\text { Related Treatment }\end{array}$ & $\begin{array}{c}\text { No. }(\%) \text { of Cases* } \\
(n=69)\end{array}$ \\
\hline \multicolumn{2}{|l|}{$\begin{array}{l}\text { Cefazolin and probenecid dose } \\
\text { and frequency }\end{array}$} \\
\hline $\begin{array}{l}\text { Cefazolin } 2 \text { g IV bid with probenecid } \\
1 \text { g PO bid }\end{array}$ & (97) \\
\hline $\begin{array}{l}\text { Cefazolin } 1 \mathrm{~g} \text { IV bid with probenecid } \\
1 \mathrm{~g} \text { PO bid }\end{array}$ & (1) \\
\hline $\begin{array}{l}\text { Cefazolin } 2 \text { g IV daily with probenecid } \\
1 \text { g PO daily }\end{array}$ & (1) \\
\hline \multicolumn{2}{|l|}{$\begin{array}{l}\text { Duration of cefazolin and probenecid } \\
\text { therapy (days) (mean } \pm \text { SD) }\end{array}$} \\
\hline All diagnoses & $36.0 \pm 16.1$ \\
\hline Prosthetic joint infection & $42.9 \pm 22.9$ \\
\hline Osteomyelitis & $33.7 \pm 11.8$ \\
\hline Septic arthritis & $23.2 \pm 13.3$ \\
\hline Diskitis/vertebral osteomyelitis & $36.4 \pm 12.2$ \\
\hline $\begin{array}{l}\text { Duration of IV antibiotic therapy before } \\
\text { cefazolin and probenecid (days) } \\
\text { (mean } \pm \text { SD) }\end{array}$ & $11.0 \pm 10.0$ \\
\hline $\begin{array}{l}\text { Antimicrobial therapy related to bone or } \\
\text { joint infection, concurrent with } \\
\text { cefazolin and probenecid }\end{array}$ & 9 \\
\hline Rifampin & (7) \\
\hline Metronidazole & (4) \\
\hline Ciprofloxacin & (1) \\
\hline
\end{tabular}

without sustained resolution up to 12 months, 2 involved prosthetic joint infections and 2 involved osteomyelitis. Initial resolution was not achieved in $16(23 \%)$ of the patient cases, and sustained resolution was not achieved in 20 (29\%) of all patient cases. Time from the start of any IV antimicrobial therapy to initial documented resolution was 89.2 (SD 46.4) days.

Readmission to hospital related to infection was documented in $11(16 \%)$ of the patient cases. Seven cases required readmission during treatment, and 4 required readmission after completion of the prescribed course of cefazolin and probenecid. Recurrence of infection was documented in 6 (9\%) of the patient cases. Treatment failure requiring a change in therapy at any time during the initial month after completion of the cefazolin and probenecid treatment occurred in 7 patient cases (10\%).

Five of the patients in this study died. Three of these patients were receiving palliative care at the time of admission, all because of malignancy. For the fourth patient, palliation was started approximately 6 months after the end of treatment with cefazolin and probenecid, also because of malignancy. For the fifth patient, the goal of therapy was transitioned from cure to palliation of symptoms within 6 months of treatment completion with cefazolin and probenecid. This patient had multiple comorbidities, including severe peripheral vascular disease with several chronic ulcers, chronic heart failure, diabetes mellitus, and chronic obstructive pulmonary disease.
Table 3. Outcomes and Related Data

\begin{tabular}{|c|c|c|}
\hline Outcome & $\begin{array}{l}\text { No. }(\%) \\
(n=\end{array}$ & $\begin{array}{l}\text { f Cases* } \\
69)\end{array}$ \\
\hline Completion of cefazolin and probenecid & & \\
\hline Yes & 56 & $(81)$ \\
\hline No & 13 & (19) \\
\hline Adverse drug reaction & 3 & (4) \\
\hline Death & 1 & (1) \\
\hline Non-susceptible organism & 1 & (1) \\
\hline $\begin{array}{l}\text { Refusal of twice-daily treatment after start } \\
\text { of therapy }\end{array}$ & 1 & (1) \\
\hline Readmission, recurrence & 7 & $(10)$ \\
\hline Initial resolution of infectious signs and symptoms & 53 & $(77)$ \\
\hline Prosthetic joint infection $(n=18)$ & 16 & (23) \\
\hline Osteomyelitis $(n=29)$ & 23 & (33) \\
\hline Septic arthritis $(n=5)$ & 3 & (4) \\
\hline Diskitis/vertebral osteomyelitis ( $n=17$ ) & 11 & $(16)$ \\
\hline No resolution & & \\
\hline Readmission, recurrence, treatment failure & 7 & $(10)$ \\
\hline Adverse drug reaction & 3 & (4) \\
\hline Palliative at admission & 3 & (4) \\
\hline Refusal of twice-daily treatment & 1 & (1) \\
\hline Noncompliance, IV drug use & 1 & (1) \\
\hline Non-susceptible organism & 1 & (1) \\
\hline $\begin{array}{l}\text { Sustained resolution of infectious signs and } \\
\text { symptoms at } 12 \text { months }\end{array}$ & & \\
\hline Yes & 49 & (71) \\
\hline No & & \\
\hline $\begin{array}{l}\text { Receiving palliative care within } 12 \text { months } \\
\text { after treatment }\end{array}$ & 2 & (3) \\
\hline Readmission, recurrence & 2 & (3) \\
\hline $\begin{array}{l}\text { Time to achieve documented resolution } \neq \\
\text { (days) (mean } \pm \text { SD) }\end{array}$ & 89.2 & 46.4 \\
\hline $\begin{array}{l}\text { Readmitted to hospital for antimicrobial } \\
\text { therapy related to initial infection }\end{array}$ & 11 & $(16)$ \\
\hline Treatment failure requiring change in therapy & 7 & $(10)$ \\
\hline Died & 5 & $(7)$ \\
\hline Receiving palliative care at time of admission & 3 & (4) \\
\hline Step-down from IV to oral antibiotics & 39 & $(57)$ \\
\hline To complete duration of antibiotic therapy & 33 & (48) \\
\hline Suppression of chronic infection & 6 & (9) \\
\hline Step-down by specific drug & & \\
\hline Cephalexin & 27 & (39) \\
\hline Cefuroxime & 3 & (4) \\
\hline Penicillin & 2 & (3) \\
\hline Amoxicillin-clavulanate & 2 & (3) \\
\hline Cephalexin/rifampin & 1 & (1) \\
\hline Ciprofloxacin/rifampin & 1 & (1) \\
\hline Levofloxacin/rifampin & 1 & (1) \\
\hline Ciprofloxacin/amoxicillin-clavulanate & 1 & (1) \\
\hline Amoxicillin & 1 & (1) \\
\hline $\begin{array}{l}\text { Adverse effects causing change in therapy } \\
\text { or discontinuation }\end{array}$ & & \\
\hline Any adverse effects & 3 & (4) \\
\hline Nausea/vomiting & 2 & (3) \\
\hline Clostridioides difficile & 1 & (1) \\
\hline $\begin{array}{l}\text { SD }=\text { standard deviation. } \\
\text { *Except where indicated otherwise. The } 69 \text { patien } \\
\text { a total of } 68 \text { patients; } 1 \text { patient had } 2 \text { separate co } \\
\text { that qualified for inclusion } \\
\text { +The } n \text { values in column } 1 \text { indicate the number of } \\
\text { type of infection. }\end{array}$ & $\begin{array}{l}\text { t cases } \\
\text { urses } 0 \\
\text { patien }\end{array}$ & $\begin{array}{l}\text { resented } \\
\text { erapy } \\
\text { vith each }\end{array}$ \\
\hline
\end{tabular}


Adverse effects thought to be due to cefazolin and probenecid resulted in a change of therapy in 3 patient cases. Gastrointestinal adverse effects such as nausea and vomiting were responsible for 2 of these changes in therapy, whereas the third patient experienced Clostridioides difficile infection.

Several characteristics were associated with favourable or poor outcomes. Male sex (OR 5.52, 95\% CI 1.30-33.71, $p=0.016$ ) and immunocompetence (OR 5.39, 95\% CI 0.99-32.05, $p=0.052$ ) were associated with greater likelihood of documented resolution. Those with recurrent or chronic infection had higher odds of readmission (OR 5.86, 95\% CI 1.26-31.85, $p=0.021$ ). Recurrent or chronic infection (OR 15.25, 95\% CI 1.54-771.86, $p=0.013$ ) and any history of bone or joint infection (OR 11.16, $95 \%$ CI $1.14-559.68, p=0.034$ ) were associated with greater odds of recurrence or relapsed infection.

\section{DISCUSSION}

To our knowledge, the combination of cefazolin with probenecid administered as antimicrobial therapy for bone and joint infections has not previously been described. Despite this lack of evidence, its use to facilitate IV antibiotic therapy for such infections in the outpatient setting has become common practice at our institution. We believe that this retrospective observational study is the first to describe prescribing patterns and patient outcomes associated with cefazolin and probenecid for treatment of bone and joint infections.

In more than $80 \%$ of patient cases, the clinical response to the intended course of cefazolin and probenecid was sufficient to allow clinicians to consider the treatment appropriate for discontinuing further IV antimicrobial therapy. These results suggest that the combination of cefazolin and probenecid may be a reasonable component of antimicrobial therapy for bone and joint infections and hence that further exploration in controlled studies is warranted. Seventy-nine percent of patient cases involving osteomyelitis had initial resolution of infection, which is comparable to the approximately $70 \%$ treatment success rate reported in the literature. ${ }^{16}$ In those with septic arthritis, the resolution rate was $60 \%$, also comparable to the $53 \%-69 \%$ reported in the literature. ${ }^{13}$ However, in our study, only 5 patients had septic arthritis, which makes it difficult to interpret and compare our findings for this specific population. The initialresolution rate of $89 \%$ for cases of prosthetic joint infection is at the higher end of previously reported success rates $(31 \%-90 \%){ }^{14}$ Four patient cases had initial resolution that was not sustained over the long term. For 2 of these patient cases, both involving osteomyelitis, the goal of care was transitioned, within 12 months of treatment, from cure to palliation of symptoms (one possibly related to chronic infection, one unrelated). The remaining 2 patient cases involved prosthetic joint infections, and the patients were readmitted to hospital with recurrent infection. Documented resolution was not solely related to efficacy of treatment; other factors, such as adverse drug effects or death for reasons unrelated to the infection, may have led to absence of documented resolution (Table 3).

Almost all patient cases had 1 or more risk factors associated with a bone or joint infection ( $n=66[96 \%])$, which have been linked to worse outcomes in the literature. ${ }^{10}$ Those with a history of bone or joint infection had significantly higher odds of relapse or recurrence of infection, and those with recurrent or chronic infection had significantly higher odds of relapse or recurrence of infection or readmission to hospital. These results are to be expected, given that recurrence of infection occurs frequently in this population. ${ }^{11}$

The pathogen most often identified in this study was methicillin-susceptible $S$. aureus, comparable to what has been documented in the literature. ${ }^{9,10}$ In our study, $20 \%$ of patient cases were treated empirically for the entirety of their treatment course, but the use of empiric therapy did not correlate with worse outcomes.

Concerns have been raised about the tolerability of probenecid, given that it has been associated with gastrointestinal upset, including nausea and vomiting. Only $4 \%$ of the patient cases in this study had a gastrointestinal adverse effect leading to discontinuation of therapy; in 1 patient, the cause of discontinuation was $C$. difficile, which is known to be correlated with antimicrobial therapy and not probenecid. However, there may have been selection bias in this study, given that a test dose of cefazolin and probenecid is prescribed before discharge for patients at our institution to demonstrate tolerability.

In our study, a regimen of cefazolin $2 \mathrm{~g}$ IV and probenecid $1 \mathrm{~g}$ PO, both twice daily, was prescribed for $97 \%$ of patient cases (Table 2). This regimen for cefazolin corresponds with the regimen of $2 \mathrm{~g}$ every $12 \mathrm{~h}$ suggested by the pharmacokinetic modelling of Spina and Dillon. ${ }^{17}$ These authors assessed the ability of probenecid to achieve therapeutic cefazolin serum concentrations for the treatment of cellulitis, although their modelling analyzed probenecid $500 \mathrm{mg}$ PO 4 times a day. ${ }^{17}$ Doses reported in the literature for skin and soft-tissue infections are variable, ${ }^{6,7,18}$ and our findings may be used to guide prescribing for bone and joint infections.

The limitations of this study include its retrospective and observational nature. Our analysis was limited to the data available in our databases and was reliant on the quality of documentation. Patient records were screened on the basis of available databases, which may not have captured all patients treated with this drug combination. Another limitation is the absence of an active comparator group receiving standard treatment; therefore, we compared our patient outcomes with those described in the literature.

Our results may not be generalizable to those who are more acutely ill, because the patients in this study were stable enough to be discharged home from hospital. Most of our patient cases 
were seen by an infectious diseases physician, which could have led to selection bias, with more complex cases (e.g., cases with more comorbidities, cases with chronic infections) being included in the study; less complex cases might be treated differently and might have different outcomes. Access to an infectious diseases physician may not always be feasible, and these specialists may be more comfortable with this treatment strategy than other physicians. Finally, this study had a relatively small sample size, and the results should be interpreted with caution.

Future research should involve larger prospective evaluations of this combination of medications, with comparison to the standard of care, to assess efficacy and safety of use in patients with bone and joint infections. The effectiveness of cefazolin and probenecid for other invasive infections, such as bacteremia and endocarditis, should also be evaluated.

\section{CONCLUSION}

In most patient cases in this study, a regimen of cefazolin $2 \mathrm{~g}$ IV and probenecid $1 \mathrm{~g}$ PO, both twice daily, was prescribed. The use of cefazolin and probenecid for the treatment of bone and joint infections appears to have had comparable outcomes to what has been described in the literature for standard treatment, with completion of therapy for $81 \%$ of patient cases, and an overall rate of initial documented resolution of infection of $77 \%$, sustained for 12 months in $71 \%$. The results of this small, single-centre retrospective analysis should be interpreted with caution but may be used to guide future research.

\section{References}

1. Stengal D, Bauwens K, Sehouli J, Ekkernkamp A, Porzsolt F. Systematic review and meta-analysis of antibiotic therapy for bone and joint infections. Lancet Infect Dis. 2001;1(3):175-88.

2. Gilchrist M, Seaton R. Outpatient parenteral antimicrobial therapy and antimicrobial stewardship: challenges and checklists. J Antimicrob Chemother. 2015;70(4):965-70.

3. Karney W, Turck M, Holmes K. Cefazolin in the treatment of gonorrhea. J Infect Dis. 1973;128 Suppl:S399-S403.

4. Duncan W. Treatment of gonorrhea with cefazolin plus probenecid. J Infect Dis. 1974;130(4):398-401.

5. Pulia M, Calderone M, Meister J, Santistevan J, May L. Update on management of skin and soft tissue infections in the emergency department. Curr Infect Dis Rep. 2014;16(9):418.

6. Dalen D, Fry A, Campbell SG, Eppler J, Zed PJ. Intravenous cefazolin plus oral probenecid versus oral cephalexin for the treatment of skin and soft tissue infections: a double-blind, non-inferiority, randomised controlled trial. Emerg Med J. 2018;35(8):492-8.

7. Grayson ML, McDonald M, Gibson K, Athan E, Munckhof W, Paull P, et al. Once-daily intravenous cefazolin plus oral probenecid is equivalent to once-daily intravenous ceftriaxone plus oral placebo for the treatment of moderate-to-severe cellulitis in adults. Clin Infect Dis. 2002;34(11):1440-8.

8. Calhoun J. Adult osteomyelitis. Infect Dis Clin North Am. 2006;19(4):765-86.

9. Kavanaugh N, Ryan EJ, Widaa A, Sexton G, Fennell J, O’Rourke S, et al. Staphylococcal osteomyelitis: disease progression, treatment challenges, and future directions. Clin Microbiol Rev. 2018;31(2):e00084-17.
10. Senneville E, Joulie D, Legout L, Valette M, Dezeque H, Beltrand E, et al. Outcome and predictors of treatment failure in total hip/knee prosthetic joint infections due to Staphylococcus aureus. Clin Infect Dis. 2011;53(4):334-40.

11. Conterno L, Turchi M. Antibiotics for treating chronic osteomyelitis in adults. Cochrane Database Sys Rev. 2013 6;(9):CD004439.

12. Berbari E, Kanj S, Kowalski T, Darouiche R, Widmer A, Schmitt S, et al.; Infectious Diseases Society of America. 2015 Infectious Diseases Society of America (IDSA) clinical practice guidelines for the diagnosis and treatment of native vertebral osteomyelitis in adults. Clin Infect Dis. 2015;61(6): e26-e46.

13. Ravindran V, Logan I, Bourke BE. Medical vs surgical treatment for the native joint in septic arthritis: a 6-year, single UK academic centre experience. Rheumatology. 2009;48(10):1320-2.

14. Tande A, Patel R. Prosthetic joint infection. Clin Microbiol Rev. 2014; 27(2):302-45.

15. Zeller V, Durand F, Kitzis M, Lhotellier L, Ziza J, Mamoudy P, et al. Continuous cefazolin infusion to treat bone and joint infections: clinical efficacy, feasibility, safety, and serum and bone concentrations. Antimicrob Agents Chemother. 2009;53(3):883-7.

16. Tice A, Hoaglund P, Shoultz D. Risk factors and treatment outcomes in osteomyelitis. J Antimicrob Chemother. 2003;51(5):1261-8.

17. Spina SP, Dillon EC Jr. Effect of chronic probenecid therapy on cefazolin serum concentrations. Ann Pharmacother. 2003;37(5):621-4.

18. Brown G, Zemcov S, Clarke A. Effect of probenecid on cefazolin serum concentrations. J Antimicrob Chemother. 1993;31(6):1009-11.

Marci E Dearing, RPh, BSC(Pharm), ACPR, PharmD, is with the Pharmacy Department, Nova Scotia Health Authority, Halifax, Nova Scotia.

Sarah V Burgess, RPh, BSC(Pharm), ACPR, PharmD, is with the Pharmacy Department, Nova Scotia Health Authority, and the College of Pharmacy, Faculty of Health, Dalhousie University, Halifax, Nova Scotia.

Valerie Murphy, RPh, BSc(Pharm), ACPR, is with the Pharmacy Department, Nova Scotia Health Authority, Halifax, Nova Scotia.

Samuel Campbell, MB BCh, CCFP(EM), DipPEC(SA), FCCHL, is with the Department of Emergency Medicine, Charles $V$ Keating Emergency and Trauma Centre, Central Zone, Nova Scotia Health Authority, Halifax, Nova Scotia.

Lynn Johnston, MD, MSC, FRCPC, is with the Division of Infectious Diseases, Central Zone, Nova Scotia Health Authority, Halifax, Nova Scotia.

Tasha D Ramsey, RPh, BSc(Pharm), ACPR, PharmD, is with the Pharmacy Department, Nova Scotia Health Authority, and the College of Pharmacy, Faculty of Health, Dalhousie University, Halifax, Nova Scotia.

Competing interests: None declared.

Address correspondence to:

Dr Tasha D Ramsey

Pharmacy Department

Nova Scotia Health Authority

1796 Summer Street

Halifax NS B3H 3 A7

e-mail: tramseypublications@gmail.com

Funding: None received.

Acknowledgements: The authors thank Dr Susan Bowles for her mentorship and statistical interpretation in the execution of this study (outside her role as an Associate Editor for the Canadian Journal of Hospital Pharmacy). 